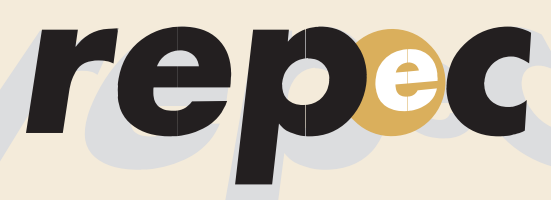

REPeC, Brasília, v. 9, n. 4, informações editoriais, p. 480-489, out./dez. 2015

Disponível online em www.repec.org.br
Revista de Educação e Pesquisa em Contabilidade Journal of Education and Research in Accounting Revista de Educación e Investigación en Contabilidad

Periódico Trimestral, digital e gratuito publicado pela Academia Brasileira de Ciências Contábeis ISSN 1981-8610

\title{
Informações Editoriais 2015
}

Artigos Publicados - 2015

\begin{tabular}{lcc}
\hline Edições & Autores por Edição & Artigos por Edição \\
\hline Jan./Mar., 2015 & 18 & 6 \\
\hline Abr./Jun., 2015 & 20 & 6 \\
\hline Jul./Set., 2015 & 21 & 6 \\
\hline Out./Dez., 2015 & 22 & 6 \\
\hline Total & 81 & 24 \\
\hline Média & 20,25 & 6 \\
\hline
\end{tabular}

Número de Artigos por Unidade de Federação - 2015

\begin{tabular}{|c|c|c|c|c|c|c|c|c|c|c|}
\hline \multirow{3}{*}{ Unidade da Federação } & \multicolumn{10}{|c|}{ Edições } \\
\hline & \multicolumn{2}{|c|}{ Jan./Mar. } & \multicolumn{2}{|c|}{ Abr./Jun. } & \multicolumn{2}{|c|}{ Jul./Set. } & \multicolumn{2}{|c|}{ Out./Dez. } & \multicolumn{2}{|c|}{ Total } \\
\hline & $\mathrm{Fi}$ & $\mathrm{Fi} \%$ & $\mathrm{Fi}$ & $\mathrm{Fi} \%$ & $\mathrm{Fi}$ & $\mathrm{Fi} \%$ & $\mathrm{Fi}$ & $\mathrm{Fi} \%$ & $\mathrm{Fi}$ & $\mathrm{Fi} \%$ \\
\hline CE & 1 & $17 \%$ & - & $0 \%$ & - & $0 \%$ & 1 & $17 \%$ & 2 & $8 \%$ \\
\hline ES & - & $0 \%$ & - & $0 \%$ & 1 & $17 \%$ & - & $0 \%$ & 1 & $4 \%$ \\
\hline ES/RS & 1 & $17 \%$ & - & $0 \%$ & - & $0 \%$ & - & $0 \%$ & 1 & $4 \%$ \\
\hline ES/TO & - & $0 \%$ & - & $0 \%$ & 1 & $17 \%$ & 1 & $17 \%$ & 2 & $8 \%$ \\
\hline MG & 1 & $17 \%$ & - & $0 \%$ & - & $0 \%$ & 1 & $17 \%$ & 2 & $8 \%$ \\
\hline MG/SP & - & $0 \%$ & - & $0 \%$ & 1 & $17 \%$ & - & $0 \%$ & 1 & $4 \%$ \\
\hline MG/SP/CE/RS & - & $0 \%$ & 1 & $17 \%$ & - & $0 \%$ & - & $0 \%$ & 1 & $4 \%$ \\
\hline MG/SP/ES & 1 & $17 \%$ & - & $0 \%$ & - & $0 \%$ & - & $0 \%$ & 1 & $4 \%$ \\
\hline PB & - & $0 \%$ & 1 & $17 \%$ & 1 & $17 \%$ & 1 & $17 \%$ & 3 & $13 \%$ \\
\hline PR & - & $0 \%$ & 1 & $17 \%$ & - & $0 \%$ & - & $0 \%$ & 1 & $4 \%$ \\
\hline $\mathrm{PR} / \mathrm{SC}$ & - & $0 \%$ & - & $0 \%$ & 1 & $17 \%$ & 1 & $17 \%$ & 2 & $8 \%$ \\
\hline $\mathrm{RJ}$ & - & $0 \%$ & 1 & $17 \%$ & - & $0 \%$ & - & $0 \%$ & 1 & $4 \%$ \\
\hline SC & - & $0 \%$ & - & $0 \%$ & - & $0 \%$ & 1 & $17 \%$ & 1 & $4 \%$ \\
\hline SC/SP & - & $0 \%$ & 1 & $17 \%$ & - & $0 \%$ & - & $0 \%$ & 1 & $8 \%$ \\
\hline SP & 1 & $17 \%$ & - & $0 \%$ & - & $0 \%$ & - & $0 \%$ & 1 & $4 \%$ \\
\hline Portugal & 1 & $17 \%$ & 1 & $17 \%$ & 1 & $17 \%$ & - & $0 \%$ & 3 & $17 \%$ \\
\hline Total & 6 & $100 \%$ & 6 & $100 \%$ & 6 & $100 \%$ & 6 & $100 \%$ & 24 & $100 \%$ \\
\hline
\end{tabular}


Número de Autores por Unidade de Federação - 2015

\begin{tabular}{|c|c|c|c|c|c|c|c|c|c|c|}
\hline \multirow{3}{*}{ Unidade da Federação } & \multicolumn{10}{|c|}{ Edições } \\
\hline & \multicolumn{2}{|c|}{ Jan./Mar. } & \multicolumn{2}{|c|}{ Abr./Jun. } & \multicolumn{2}{|c|}{ Jul./Set. } & \multicolumn{2}{|c|}{ Out./Dez. } & \multicolumn{2}{|c|}{ Total } \\
\hline & $\mathrm{Fi}$ & $\mathrm{Fi} \%$ & $\mathrm{Fi}$ & $\mathrm{Fi} \%$ & $\mathrm{Fi}$ & $\mathrm{Fi} \%$ & $\mathrm{Fi}$ & $\mathrm{Fi} \%$ & $\mathrm{Fi}$ & $\mathrm{Fi} \%$ \\
\hline Ceará & 3 & $17 \%$ & 1 & $5 \%$ & - & $0 \%$ & 5 & $23 \%$ & 9 & $11 \%$ \\
\hline Espírito Santo & 2 & $11 \%$ & - & $0 \%$ & 5 & $24 \%$ & 3 & $14 \%$ & 10 & $12 \%$ \\
\hline Minas Gerais & 4 & $22 \%$ & 1 & $5 \%$ & 2 & $10 \%$ & 5 & $23 \%$ & 12 & $15 \%$ \\
\hline Paraíba & - & $0 \%$ & 4 & $20 \%$ & 4 & $19 \%$ & 2 & $9 \%$ & 10 & $12 \%$ \\
\hline Paraná & - & $0 \%$ & 3 & $15 \%$ & 1 & $5 \%$ & 2 & $9 \%$ & 6 & $7 \%$ \\
\hline Rio de Janeiro & - & $0 \%$ & 2 & $10 \%$ & - & $0 \%$ & - & $0 \%$ & 2 & $2 \%$ \\
\hline Rio Grande do Sul & 1 & $6 \%$ & 1 & $5 \%$ & - & $0 \%$ & - & $0 \%$ & 2 & $2 \%$ \\
\hline Santa Catarina & - & $0 \%$ & 1 & $5 \%$ & 1 & $5 \%$ & 3 & $14 \%$ & 5 & $6 \%$ \\
\hline São Paulo & 4 & $22 \%$ & 5 & $25 \%$ & 3 & $14 \%$ & 1 & $5 \%$ & 13 & $16 \%$ \\
\hline Tocantins & - & $0 \%$ & - & $0 \%$ & 1 & $5 \%$ & 1 & $5 \%$ & 2 & $2 \%$ \\
\hline Portugal & 4 & $22 \%$ & 2 & $10 \%$ & 4 & $19 \%$ & - & $0 \%$ & 10 & $12 \%$ \\
\hline Total & 18 & $100 \%$ & 20 & $100 \%$ & 21 & $100 \%$ & 22 & $100 \%$ & 81 & $100 \%$ \\
\hline
\end{tabular}

Inventário dos Artigos - 2015

\begin{tabular}{lc}
\hline Itens & $\mathbf{2 0 1 4}$ \\
\hline Estoque inicial de artigos em $1^{\circ}$ de janeiro & 25 \\
\hline$\quad$ Em avaliação & 25 \\
\hline Númados & 0 \\
\hline Número de artigos submetidos & 115 \\
\hline Número de artigos reprovados aprovados no Desk Review & $(31)$ \\
\hline Número de artigos retirados & $(56)$ \\
\hline Número de artigos publicados & $(1)$ \\
\hline Número de artigos aceitos para edições seguintes & $(24)$ \\
\hline Estoque Final de artigos em avaliação em 31 de Dezembro & $(2)$ \\
\hline
\end{tabular}


Instituições de Origem dos Autores dos Artigos - 2015

\begin{tabular}{|c|c|c|c|c|c|c|}
\hline \multirow{3}{*}{$\begin{array}{l}\text { UF/IES de origem } \\
\text { CE/UFC }\end{array}$} & \multicolumn{6}{|c|}{ Edições } \\
\hline & \multirow{2}{*}{$\frac{\text { Jan./Mar. }}{3}$} & \multirow{2}{*}{$\frac{\text { Abr./Jun. }}{1}$} & \multirow[t]{2}{*}{ Jul./Set. } & \multirow{2}{*}{$\frac{\text { Out./Dez. }}{5}$} & \multicolumn{2}{|c|}{ Total } \\
\hline & & & & & 9 & $11 \%$ \\
\hline ES/FUCAPE & 2 & & & & 2 & $2 \%$ \\
\hline ES/UFES & & & 5 & 3 & 8 & $10 \%$ \\
\hline MG/UFMG & & 1 & 1 & & 2 & $2 \%$ \\
\hline MG/FUNORTE & 1 & & & & 1 & $1 \%$ \\
\hline MG/UFU & 3 & & 1 & & 4 & $5 \%$ \\
\hline MG/UFLA & & & & 4 & 4 & $5 \%$ \\
\hline MG/UFV & & & & 1 & 1 & $1 \%$ \\
\hline PR/UFPR & & & & 1 & 1 & $1 \%$ \\
\hline PR/UTFPR & & 3 & & & 3 & $4 \%$ \\
\hline PR/UEL & & & & 1 & 1 & $1 \%$ \\
\hline PR/UNICENTRO & & & 1 & & 1 & $1 \%$ \\
\hline PB/UFPB & & 4 & 4 & 1 & 9 & $11 \%$ \\
\hline PB/UNIPÊ & & & & 1 & 1 & $1 \%$ \\
\hline RJ/UFRJ & & 2 & & & 2 & $2 \%$ \\
\hline RS/UNISINOS & 1 & 1 & & & 2 & $2 \%$ \\
\hline SC/FURB & & 1 & 1 & 1 & 3 & $4 \%$ \\
\hline SC/UDESC & & & & 1 & 1 & $1 \%$ \\
\hline SC/URI & & & & 1 & 1 & $1 \%$ \\
\hline SP/USP & & 2 & 3 & 1 & 6 & $7 \%$ \\
\hline SP/USPRP & 1 & & & & 1 & $1 \%$ \\
\hline SP/UPM & 3 & & & & 3 & $4 \%$ \\
\hline SP/FECAP & & 3 & & & 3 & $4 \%$ \\
\hline TO/IFTO & & & 1 & 1 & 2 & $2 \%$ \\
\hline PORTUGAL/ISCAL & 3 & & 3 & & 6 & $7 \%$ \\
\hline PORTUGAL/UAL & 1 & & 1 & & 2 & $2 \%$ \\
\hline PORTUGAL/UA & & 2 & & & 2 & $2 \%$ \\
\hline TOTAL & 18 & 20 & 21 & 22 & 81 & $100 \%$ \\
\hline
\end{tabular}




\section{Periodo Médio de Circulação dos Artigos Publicados - 2015}

Vol. 9, n. 1, 2015

\begin{tabular}{|c|c|c|c|c|c|c|c|c|c|}
\hline $\begin{array}{l}\mathrm{N}^{\circ} \text {. do } \\
\text { Artigo }\end{array}$ & $\begin{array}{c}\text { Data de } \\
\text { submissão }\end{array}$ & $\begin{array}{l}\text { Avaliado } \\
\text { pelos } \\
\text { Pares }\end{array}$ & $\begin{array}{l}\text { Reformulado } \\
\text { pelos autores }\end{array}$ & $\begin{array}{c}\text { Data da } \\
\text { Aceitação }\end{array}$ & $\begin{array}{c}\text { Data da } \\
\text { Publicação }\end{array}$ & $\begin{array}{c}\mathbf{N}^{\circ} \text {. de } \\
\text { dias para } \\
\text { avaliação }\end{array}$ & $\begin{array}{c}\mathbf{N}^{\circ} \text {. de } \\
\text { dias para } \\
\text { reformulação }\end{array}$ & $\begin{array}{c}N^{\circ} \text {. de } \\
\text { dias para } \\
\text { recomendação } \\
\text { de publicação }\end{array}$ & $\begin{array}{c}\mathrm{N}^{\circ} \text {. de dias } \\
\text { entre a } \\
\text { submissão e } \\
\text { publicação }\end{array}$ \\
\hline 1 & $28 / 04 / 2014$ & $16 / 09 / 2014$ & $22 / 09 / 2014$ & $16 / 01 / 2015$ & $31 / 03 / 2015$ & 138 & 6 & 114 & 333 \\
\hline 2 & 27/10/2014 & 08/01/2015 & $30 / 01 / 2015$ & $02 / 02 / 2015$ & $31 / 03 / 2015$ & 71 & 22 & 2 & 154 \\
\hline 3 & $14 / 11 / 2014$ & 08/01/2015 & $02 / 02 / 2015$ & 05/02/2015 & $31 / 03 / 2015$ & 54 & 24 & 3 & 137 \\
\hline 4 & 08/10/2014 & $29 / 11 / 2014$ & $22 / 12 / 2014$ & $02 / 02 / 2015$ & $31 / 03 / 2015$ & 51 & 23 & 40 & 173 \\
\hline 5 & $21 / 04 / 2014$ & $21 / 01 / 2015$ & 29/01/2015 & $31 / 01 / 2015$ & $31 / 03 / 2015$ & 270 & 8 & 2 & 340 \\
\hline 6 & $18 / 11 / 2014$ & $02 / 02 / 2015$ & 03/02/2015 & 05/02/2015 & $31 / 03 / 2015$ & 74 & 1 & 2 & 133 \\
\hline \multicolumn{6}{|c|}{ Média de dias para publicação de artigos por Edição } & 110 & & & 212 \\
\hline \multicolumn{6}{|c|}{ Média de meses para publicação de artigos por Edição } & 3,7 & & & 7,1 \\
\hline
\end{tabular}

Vol. 9, n. 2, 2015

\begin{tabular}{|c|c|c|c|c|c|c|c|c|c|}
\hline $\begin{array}{l}N^{\circ} \text {. do } \\
\text { Artigo }\end{array}$ & $\begin{array}{c}\text { Data de } \\
\text { submissão }\end{array}$ & $\begin{array}{l}\text { Avaliado } \\
\text { pelos } \\
\text { Pares }\end{array}$ & $\begin{array}{l}\text { Reformulado } \\
\text { pelos autores }\end{array}$ & $\begin{array}{c}\text { Data da } \\
\text { Aceitação }\end{array}$ & $\begin{array}{c}\text { Data da } \\
\text { Publicação }\end{array}$ & $\begin{array}{c}N^{\circ} \text {. de } \\
\text { dias para } \\
\text { avaliação }\end{array}$ & $\begin{array}{c}N^{\circ} \text {. de } \\
\text { dias para } \\
\text { reformulação }\end{array}$ & $\begin{array}{c}\mathbf{N}^{\circ} \text {. de } \\
\text { dias para } \\
\text { recomendação } \\
\text { de publicação }\end{array}$ & $\begin{array}{c}\mathrm{N}^{\circ} \text {. de dias } \\
\text { entre a } \\
\text { submissão e } \\
\text { publicação }\end{array}$ \\
\hline 1 & 04/08/2014 & $30 / 01 / 2015$ & $25 / 02 / 2015$ & $14 / 04 / 2015$ & $30 / 06 / 2015$ & 176 & 25 & 49 & 326 \\
\hline 2 & $19 / 11 / 2014$ & $14 / 03 / 2015$ & 20/03/2015 & $03 / 05 / 2015$ & $30 / 06 / 2015$ & 115 & 6 & 43 & 221 \\
\hline 3 & 07/01/2015 & $27 / 02 / 2015$ & 17/03/2015 & $21 / 03 / 2015$ & $30 / 06 / 2015$ & 50 & 20 & 4 & 173 \\
\hline 4 & $04 / 11 / 2014$ & $20 / 02 / 2015$ & $28 / 02 / 2015$ & 06/03/2015 & $30 / 06 / 2015$ & 106 & 8 & 6 & 236 \\
\hline 5 & $07 / 11 / 2014$ & $16 / 02 / 2015$ & $13 / 03 / 2015$ & $17 / 03 / 2015$ & $30 / 06 / 2015$ & 99 & 27 & 4 & 233 \\
\hline 6 & $11 / 03 / 2015$ & $17 / 05 / 2015$ & $11 / 06 / 2015$ & $11 / 06 / 2015$ & $30 / 06 / 2015$ & 66 & 24 & 0 & 109 \\
\hline \multicolumn{6}{|c|}{ Média de dias para publicação de artigos por Edição } & 102 & & & 216 \\
\hline \multicolumn{6}{|c|}{ Média de meses para publicação de artigos por Edição } & 3,4 & & & 7,2 \\
\hline
\end{tabular}


Vol. 9, n. 3, 2015

\begin{tabular}{|c|c|c|c|c|c|c|c|c|c|}
\hline $\begin{array}{l}N^{\circ} \text {. do } \\
\text { Artigo }\end{array}$ & $\begin{array}{c}\text { Data de } \\
\text { submissão }\end{array}$ & $\begin{array}{l}\text { Avaliado } \\
\text { pelos } \\
\text { Pares }\end{array}$ & $\begin{array}{l}\text { Reformulado } \\
\text { pelos autores }\end{array}$ & $\begin{array}{c}\text { Data da } \\
\text { Aceitação }\end{array}$ & $\begin{array}{c}\text { Data da } \\
\text { Publicação }\end{array}$ & $\begin{array}{c}\mathrm{N}^{\circ} \text {. de } \\
\text { dias para } \\
\text { avaliação }\end{array}$ & $\begin{array}{c}\mathrm{N}^{\circ} \text {. de } \\
\text { dias para } \\
\text { reformulação }\end{array}$ & $\begin{array}{c}\mathrm{N}^{\circ} \text {. de } \\
\text { dias para } \\
\text { recomendação } \\
\text { de publicação }\end{array}$ & $\begin{array}{c}\mathrm{N}^{\circ} \text {. de dias } \\
\text { entre a } \\
\text { submissão e } \\
\text { publicação }\end{array}$ \\
\hline 1 & $03 / 02 / 2014$ & $16 / 03 / 2015$ & 05/04/2015 & 08/04/2015 & $30 / 09 / 2015$ & 403 & 19 & 3 & 597 \\
\hline 2 & $31 / 03 / 2015$ & 06/07/2015 & 27/07/2015 & 04/08/2015 & $30 / 09 / 2015$ & 96 & 21 & 7 & 180 \\
\hline 3 & $17 / 02 / 2015$ & 23/03/2015 & $22 / 04 / 2015$ & $10 / 08 / 2015$ & $30 / 09 / 2015$ & 36 & 29 & 108 & 223 \\
\hline 4 & $10 / 03 / 2015$ & $24 / 05 / 2015$ & 01/07/2015 & 04/08/2015 & $30 / 09 / 2015$ & 74 & 37 & 33 & 200 \\
\hline 5 & $22 / 06 / 2015$ & 04/08/2015 & $18 / 06 / 2015$ & $18 / 08 / 2015$ & $30 / 09 / 2015$ & 42 & -46 & 60 & 98 \\
\hline 6 & $08 / 11 / 2014$ & $17 / 05 / 2015$ & $21 / 06 / 2015$ & 02/07/2015 & $30 / 09 / 2015$ & 189 & 34 & 11 & 322 \\
\hline \multicolumn{6}{|c|}{ Média de dias para publicação de artigos por Edição } & 140 & & & 270 \\
\hline \multicolumn{6}{|c|}{ Média de meses para publicação de artigos por Edição } & 4,7 & & & 9,0 \\
\hline
\end{tabular}

Vol. 9, n. 4, 2015

\begin{tabular}{|c|c|c|c|c|c|c|c|c|c|}
\hline $\begin{array}{l}\mathrm{N}^{\circ} \text {. do } \\
\text { Artigo }\end{array}$ & $\begin{array}{c}\text { Data de } \\
\text { submissão }\end{array}$ & $\begin{array}{l}\text { Avaliado } \\
\text { pelos } \\
\text { Pares }\end{array}$ & $\begin{array}{l}\text { Reformulado } \\
\text { pelos autores }\end{array}$ & $\begin{array}{c}\text { Data da } \\
\text { Aceitação }\end{array}$ & $\begin{array}{c}\text { Data da } \\
\text { Publicação }\end{array}$ & $\begin{array}{c}N^{\circ} \text {. de } \\
\text { dias para } \\
\text { avaliação }\end{array}$ & $\begin{array}{c}\mathbf{N}^{\circ} \text {. de } \\
\text { dias para } \\
\text { reformulação }\end{array}$ & $\begin{array}{c}\mathrm{N}^{\circ} \text {. de } \\
\text { dias para } \\
\text { recomendação } \\
\text { de publicação }\end{array}$ & $\begin{array}{c}\mathrm{N}^{\circ} \text {. de dias } \\
\text { entre a } \\
\text { submissão e } \\
\text { publicação }\end{array}$ \\
\hline 1 & $25 / 07 / 2015$ & $23 / 11 / 2015$ & $06 / 12 / 2015$ & $07 / 12 / 2015$ & $30 / 12 / 2015$ & 118 & 13 & 1 & 155 \\
\hline 2 & 03/08/2015 & $01 / 11 / 2015$ & $20 / 11 / 2015$ & $21 / 11 / 2015$ & $30 / 12 / 2015$ & 88 & 19 & 1 & 147 \\
\hline 3 & $24 / 08 / 2015$ & $18 / 11 / 2015$ & 10/12/2015 & $16 / 12 / 2015$ & $30 / 12 / 2015$ & 84 & 22 & 6 & 126 \\
\hline 4 & $16 / 03 / 2015$ & $10 / 10 / 2015$ & 28/10/2015 & $31 / 10 / 2015$ & $30 / 12 / 2015$ & 204 & 18 & 3 & 284 \\
\hline 5 & $30 / 09 / 2015$ & $31 / 10 / 2015$ & 20/11/2015 & $21 / 11 / 2015$ & $30 / 12 / 2015$ & 30 & 20 & 1 & 90 \\
\hline 6 & 06/01/2015 & $17 / 08 / 2015$ & $08 / 11 / 2015$ & $15 / 11 / 2015$ & $30 / 12 / 2015$ & 221 & 81 & 7 & 354 \\
\hline \multicolumn{6}{|c|}{ Média de dias para publicação de artigos por Edição } & 124 & & & 193 \\
\hline \multicolumn{6}{|c|}{ Média de meses para publicação de artigos por Edição } & 4,1 & & & 6,4 \\
\hline
\end{tabular}

\begin{tabular}{lcc}
\hline Média de dias para publicação de artigos por Edição em 2015 & 119,0 & 222,7 \\
\hline Média de meses para publicação de artigos por Edição em 2015 & 4,0 & 7,4 \\
\hline
\end{tabular}




\section{Avaliadores Ad Hoc e Instituição de Vínculo - 2015}

\begin{tabular}{|c|c|}
\hline Avaliador & Instituição \\
\hline Alessandra Vasconcelos Gallon & Universidade Federal do Ceará \\
\hline Adriana Maria Procópio de Araujo & Universidade de São Paulo - Ribeirão Preto \\
\hline Adriano Rodrigues & Universidade Federal do Rio de Janeiro \\
\hline Alfredo Sarlo Neto & Universidade Federal do Espírito Santo \\
\hline Alvaro Augusto Ricardino Filho & Pontifícia Universidade Católica de São Paulo \\
\hline Amaury José Rezende & Universidade de São Paulo - Ribeirão Preto \\
\hline Ana Claudia Afra Neitzke & Universidade Federal do Paraná \\
\hline Ana Cristina de Faria & Universidade Municipal de São Caetano do Sul \\
\hline Ana Maria Roux César & Universidade Presbiteriana Mackenzie \\
\hline André Carlos Busaneli De Aquino & Universidade de São Paulo - Ribeirão Preto \\
\hline Andson Braga De Aguiar & Universidade de São Paulo \\
\hline Aneide Oliveira Araujo & Universidade Federal do Rio Grande do Norte \\
\hline Antonio Carlos Dias Coelho & Universidade Federal do Ceará \\
\hline Antonio Lopo Martinez & Fucape Business School \\
\hline Aridelmo José Campanharo Teixeira & Fucape Business School \\
\hline Arilda Magda Campagnaro Teixeira & Fucape Business School \\
\hline Artur Roberto do Nascimento & Universidade Estadual de Feira de Santana \\
\hline Cacilda Andrade & Universidade Federal de Pernambuco \\
\hline Carlos Alberto Diehl & Universidade do Vale do Rio dos Sinos \\
\hline Carlos Eduardo Facin Lavarda & Fundação Universidade Regional de Blumenau \\
\hline Carlos Renato Theóphilo & Universidade Estadual de Montes Claros \\
\hline Carlos Roberto Godoy & Universidade de São Paulo - Ribeirão Preto \\
\hline César Augusto Tiburcio Silva & Universidade de Brasília \\
\hline Claudio de Souza Miranda & Universidade de São Paulo - Ribeirão Preto \\
\hline Deisy Cristina Corrêa Igarashi & Universidade Estadual do Oeste do Paraná \\
\hline Delci Grapégia Dal Vesco & Universidade Estadual do Oeste do Paraná \\
\hline Denizar Leal & Universidade Federal do Espírito Santo \\
\hline Dione Olesczuk Soutes & Universidade Estadual do Oeste do Paraná \\
\hline Edgard B. Cornachione Jr & Universidade de São Paulo \\
\hline Edilene Santos Santana & Fundação Getúlio Vargas - São Paulo \\
\hline Edílson Paulo & Universidade Federal da Paraíba \\
\hline Edvalda Araujo Leal & Universidade Federal de Uberlândia \\
\hline Elionor Farah Jreige Weffort & Fundação Escola de Comércio Álvares Penteado \\
\hline Eliseu Martins & Universidade de São Paulo \\
\hline Eric Aversari Martins & Universidade de São Paulo \\
\hline
\end{tabular}




\begin{tabular}{|c|c|}
\hline Ernani Ott & Universidade do Vale do Rio dos Sinos \\
\hline Fábio Frezatti & Universidade de São Paulo \\
\hline Fábio Moraes da Costa & Fucape Business School \\
\hline Felipe Ramos Ferreira & Fucape Business School \\
\hline Fernanda Filgueiras Sauerbronn & Universidade Federal do Rio de Janeiro \\
\hline Fernando Caio Galdi & Fucape Business School \\
\hline Fernando Dal-Ri Murcia & Universidade Federal de Santa Catarina \\
\hline Flávia Zóboli Dalmácio & Universidade de São Paulo \\
\hline Francisco Antonio Bezerra & Fundação Universidade Regional de Blumenau \\
\hline Gabriel Moreira Campos & Universidade Federal do Espírito Santo \\
\hline Gerlando Augusto Sampaio Franco de Lima & Universidade de São Paulo \\
\hline Guillermo Braunbeck & Universidade de São Paulo \\
\hline Henrique Formigoni & Universidade de São Paulo \\
\hline Ilse Maria Beuren & Universidade Federal do Paraná \\
\hline Irineu Afonso Frey & Universidade Federal de Santa Catarina \\
\hline Ivam Ricardo Peleias & Fundação Escola de Comércio Álvares Penteado \\
\hline Jacqueline Veneroso Alves da Cunha & Universidade Federal de Minas Gerais \\
\hline Jaime Crozatti & Universidade de São Paulo \\
\hline Jeronymo José Libonati & Universidade Federal de Pernanbuco \\
\hline Joanilia Neide de Sales Cia & Universidade de São Paulo \\
\hline Jorge de Souza Bispo & Receita Federal do Brasil \\
\hline Jorge Eduardo Scarpin & Universidade Federal do Paraná \\
\hline Jorge Katsumi Niyama & Universidade de Brasilia \\
\hline José Alonso Borba & Universidade Federal de Santa Catarina \\
\hline José Dionísio Gomes Da Silva & Universidade Federal do Rio Grande do Norte \\
\hline José Dutra Oliveira Neto & Universidade de São Paulo - Ribeirão Preto \\
\hline José Ellias Feres de Almeida & Universidade Federal do Espírito santo \\
\hline José Maria Dias Filho & Universidade Federal da Bahia \\
\hline Laura Calixto & Universidade Federal de São Paulo \\
\hline Lauro Brito de Almeida & Universidade Federal do Paraná \\
\hline Luis Eduardo Afonso & Universidade de São Paulo \\
\hline Luís Lima Santos & Instituto Politécnico de Leiria (IPL) - Portugal \\
\hline Luiz Alberton & Universidade Federal de Santa Catarina \\
\hline Maisa de Souza Ribeiro & Universidade de São Paulo - Ribeirão Preto \\
\hline Marcelle Colares Oliveira & Universidade Federal do Ceará \\
\hline Marcelo Alvaro da Silva Macedo & Universidade Federal do Rio de Janeiro \\
\hline Marcelo Sanches Pagliarussi & Universidade de São Paulo - Ribeirão Preto \\
\hline
\end{tabular}




\begin{tabular}{|c|c|}
\hline Márcia Maria dos Santos Bortolocci Espejo & Universidade Federal do Paraná \\
\hline Márcia Martins Mendes De Luca & Universidade Federal do Ceará \\
\hline Marcos Antonio de Souza & Universidade do Vale do Rio dos Sinos \\
\hline Marcus Vinicius Veras Machado & Universidade Federal do Ceará \\
\hline Maria José Carvalho de Souza Domingues & Fundação Universidade Regional de Blumenau \\
\hline Maria Thereza Pompa Antunes & Fipecafi \\
\hline Marta Cristina Pelucio Grecco & Universidade Presbiteriana Mackenzie \\
\hline Moacir Moacir Sancovschi & Universidade Federal do Rio de Janeiro \\
\hline Monica Sionara Schpallir Calijuri & Universidade Presbiteriana Mackenzie \\
\hline Otávio Ribeiro de Medeiros & Universidade de Brasília \\
\hline Patricia Maria Bortolon & Universidade Federal do Espírito santo \\
\hline Paulo Roberto Barbosa Lustosa & Universidade de Brasília \\
\hline Paulo Roberto da Cunha & Fundação Universidade Regional de Blumenau \\
\hline Paulo Rogério Faustino Matos & Universidade Federal do Ceará \\
\hline Raimundo Nonato Lima Filho & Universidade do Estado da Bahia \\
\hline Reinaldo Guerreiro & Universidade de São Paulo \\
\hline Reinaldo Rodrigues Camacho & Universidade Estadual de Maringá \\
\hline Renê Coppe Pimentel & Fucape Business School \\
\hline Rodrigo de Souza Gonçalves & Universidade de Brasília \\
\hline Rogério João Lunkes & Universidade Federal de Santa Catarina \\
\hline Romildo de Oliveira Moraes & Universidade Estadual de Maringá \\
\hline Romualdo Douglas Colauto & Universidade Federal do Paraná \\
\hline Sandra Maria dos Santos & Universidade Federal do Ceará \\
\hline Sérgio de ludícibus & Pontifícia Universidade Católica de São Paulo \\
\hline Silvia Pereira De Castro Casa Nova & Universidade de São Paulo \\
\hline Silvio Hiroshi Nakao & Universidade de São Paulo - Ribeirão Preto \\
\hline Solange Garcia dos Reis & Universidade de São Paulo - Ribeirão Preto \\
\hline Sonia Maria da Silva Gomes & Universidade Federal da Bahia \\
\hline Umbelina Cravo Texeira Lagioia & Universidade Federal de Pernambuco \\
\hline Valcemiro Nossa & Fucape Business School \\
\hline Vera Maria Rodrigues Ponte & Universidade Federal do Ceará \\
\hline Victor Branco de Holanda & Universidade Federal do Rio Grande do Norte \\
\hline Vilma Geni Slomski & Fundação Escola de Comércio Álvares Penteado \\
\hline Vinicius Aversari Martins & Universidade de São Paulo - Ribeirão Preto \\
\hline Wagner Moura Lamounier & Universidade Federal de Minas Gerais \\
\hline Wellington Rocha & Universidade de São Paulo \\
\hline Zaina Said El Hajj & Universidade Federal do Rio de Janeiro \\
\hline
\end{tabular}


Origem dos Avaliadores - 2015

\begin{tabular}{lcc}
\hline Estado & Quantidade & Percentual \\
\hline Bahia & 4 & $3,7 \%$ \\
\hline Ceará & 8 & $7,3 \%$ \\
\hline Distrito Federal & 5 & $4,6 \%$ \\
\hline Espirito Santo & 13 & $11,9 \%$ \\
\hline Minas Gerais & 4 & $3,7 \%$ \\
\hline Paraíba & 1 & $0,9 \%$ \\
\hline Pernambuco & 3 & $2,8 \%$ \\
\hline Paraná & 11 & $10,1 \%$ \\
\hline Rio de Janeiro & 5 & $4,6 \%$ \\
\hline Rio Grande do Norte & $2,8 \%$ \\
\hline Rio Grande do Sul & 3 & $2,8 \%$ \\
\hline Santa Catarina & 3 & $8,3 \%$ \\
\hline São Paulo & 9 & $35,8 \%$ \\
\hline Portugal & 39 & $0,9 \%$ \\
\hline Total & 1 & $100 \%$ \\
\hline
\end{tabular}


Instituições de Lotação dos Avaliadores - 2015

\begin{tabular}{|c|c|c|c|}
\hline Instituição & Estado & Quantidade & Percentual \\
\hline Universidade Estadual de Feira de Santana & BA & 1 & $0,9 \%$ \\
\hline Universidade Federal da Bahia & BA & 2 & $1,8 \%$ \\
\hline Universidade do Estado da Bahia & BA & 1 & $0,9 \%$ \\
\hline Universidade Federal do Ceará & CE & 8 & $7,3 \%$ \\
\hline Universidade de Brasília & DF & 5 & $4,6 \%$ \\
\hline Fucape Business School & ES & 8 & $7,3 \%$ \\
\hline Universidade Federal do Espírito Santo & ES & 5 & $4,6 \%$ \\
\hline Universidade Estadual de Montes Claros & MG & 1 & $0,9 \%$ \\
\hline Universidade Federal de Minas Gerais & MG & 2 & $1,8 \%$ \\
\hline Universidade Federal de Uberlândia & MG & 1 & $0,9 \%$ \\
\hline Universidade Federal da Paraíba & PB & 1 & $0,9 \%$ \\
\hline Universidade Federal de Pernambuco & PE & 3 & $2,8 \%$ \\
\hline Universidade Estadual de Maringá & PR & 2 & $1,8 \%$ \\
\hline Universidade Estadual do Oeste do Paraná & PR & 3 & $2,8 \%$ \\
\hline Universidade Federal do Paraná & PR & 6 & $5,5 \%$ \\
\hline Universidade Federal do Rio de Janeiro & $\mathrm{RJ}$ & 5 & $4,6 \%$ \\
\hline Universidade Federal do Rio Grande do Norte & $\mathrm{RN}$ & 3 & $2,8 \%$ \\
\hline Universidade do Vale do Rio dos Sinos & RS & 3 & $2,8 \%$ \\
\hline Fundação Universidade Regional de Blumenau & SC & 4 & $3,7 \%$ \\
\hline Universidade Federal de Santa Catarina & SC & 5 & $4,6 \%$ \\
\hline Faculdade Fipecafi & $\mathrm{SP}$ & 1 & $0,9 \%$ \\
\hline Fundação Escola de Comércio Álvares Penteado & $\mathrm{SP}$ & 3 & $2,8 \%$ \\
\hline Fundação Getúlio Vargas - SP & SP & 1 & $0,9 \%$ \\
\hline Pontifícia Universidade Católica de São Paulo & SP & 2 & $1,8 \%$ \\
\hline Receita Federal do Brasil & SP & 1 & $0,9 \%$ \\
\hline Universidade de São Paulo & $\mathrm{SP}$ & 15 & $13,8 \%$ \\
\hline Universidade de São Paulo de Ribeirão Preto & $\mathrm{SP}$ & 11 & $10,1 \%$ \\
\hline Universidade Federal de São Paulo & $\mathrm{SP}$ & 1 & $0,9 \%$ \\
\hline Universidade Municipal de São Caetano do Sul & SP & 1 & $0,9 \%$ \\
\hline Universidade Presbiteriana Mackenzie & SP & 3 & $2,8 \%$ \\
\hline Instituto Politécnico de Leiria (IPL) & Portugal & 1 & $0,9 \%$ \\
\hline Total & & 109 & $100 \%$ \\
\hline
\end{tabular}

